A 60-year-old man presented with worsening right upper quadrant pain, diarrhea and weight loss. Computerized tomography revealed multiple lesions in the liver that were suspicious for metastatic disease (Figure $\mathbf{1}$ ). In search of a primary lesion, colonoscopy was performed. Two rectal polyps were found and removed with snare cautery. One of the polyps $(0.9 \mathrm{~cm})$ showed a poorly differentiated neuroendocrine carcinoma $(0.3 \mathrm{~cm})$, arising in a tubulovillous adenoma, invading the muscularis mucosae but not the submucosa (Figure 2). Multiple step sections, exhausting almost all the tissue, were examined, looking for deeper invasion, but none was identified. Although cautery artifact was present at the deep edge of the specimen, it was not severe enough to warrant concern about any destruction of evidence of submucosal tumor. Immunohistochemically, the tumor was positive for neuron-specific enolase and synaptophysin but negative for chromogranin. Positivity for two out of three neuroendocrine markers supported the probability of the neuroendocrine nature of the tumor. Fine-needle aspiration of the liver lesions showed cells similar to the rectal primary. The liver tumor showed an immunohistochemical staining pattern identical to that of the rectal tumor, being positive for neuronspecific enolase and synaptophysin but negative for chromogranin. An extensive radiological work-up, including positron emission tomography, was negative for any other alternative primary lesions. The patient declined therapy and was discharged to hospice care.

Tumor was found at only two sites in this patient (rectum and liver). Since these two lesions were morphologically and immunohistochemically identical, we concluded that the liver lesions represented metastases from the rectum. The absence of other lesions radiologically and endoscopically makes it unlikely that both the rectal and hepatic lesions represent metastasis from a third, occult site.

\title{
Small Advanced Neuroendocrine Carcinoma of Rectum Discovered in an Adenomatous Polyp
}

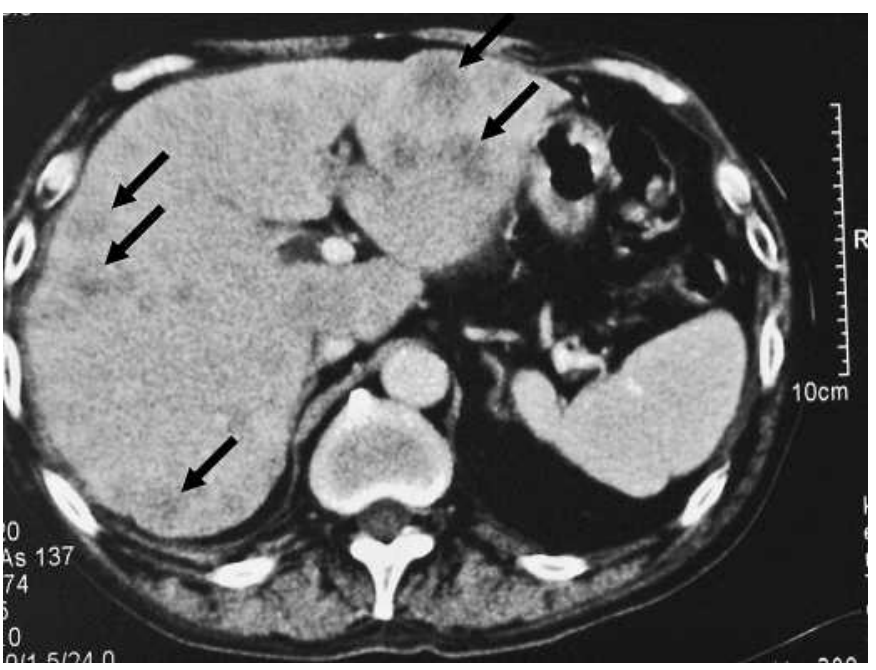

Figure 1 Computerized tomogram of abdomen showing multiple hepatic metastases (arrows).

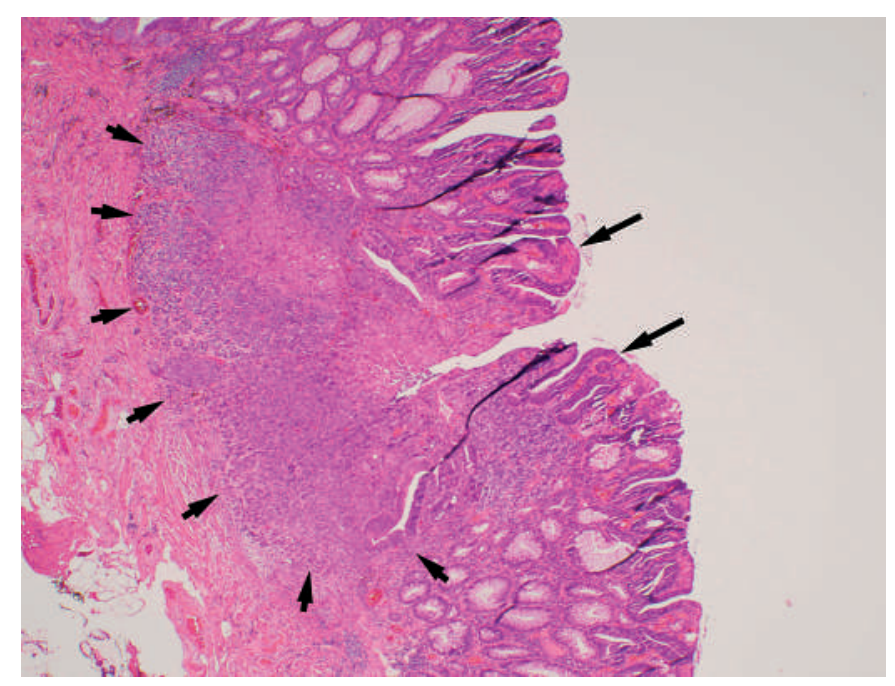

Figure 2 Histological examination showed a carcinoma (short arrows) arising within an adenomatous polyp (long arrows). Note that the submucosa is not infiltrated by tumor (hematoxylin and eosin; original magnification $\times 40$ )

Although the patient's presenting complaints were most likely related to metastatic tumor, the cause for the mild diarrhea was not readily apparent. In accordance with the patient's wishes, further work-up was not carried out, and serum gastrin or vasoactive intestinal peptide levels were not determined. In the absence of this information, the possibility that the diarrhea may have been related to hormonal products of the neuroendocrine tumor must remain speculative.

Poorly differentiated neuroendocrine carcinomas of the gastrointestinal tract carry a worse prognosis than adenocarcinomas, typically metastasizing despite only superficial invasion [2]. They often arise in the setting of an overlying adenoma, as seen in the present case [2,3]. However, our case is unique because it is an exception to the rule that colorectal tumors that do not infiltrate the submucosa are without metastatic potential [3]. Hepatic metastases in the absence of submucosal invasion can be explained by invasion of capillaries rather than lymphatics [3]. Small advanced colorectal carcinomas are rare. The smallest tumor in a series published in an earlier issue of Endoscopy was $0.8 \mathrm{~cm}$ in size [4]. A $0.3-\mathrm{cm}$ nonmetastatic neuroendocrine carcinoma arising in a tu- 
bulovillous adenoma has also been described [2]. Minamoto et al. described a 0.5 -cm nonpolypoid adenocarcinoma metastatic to lymph node [5]. To the best of our knowledge, the present case represents the smallest and most superficial metastatic colorectal carcinoma described in the literature to date. Histological examination of endoscopically "benign" polyps may reveal an unsuspected primary source of a metastatic carcinoma of unknown origin.

\section{S. Mukhopadhyay ${ }^{1}$, A. Marhaba ${ }^{2}$,} J. S. Sidhu ${ }^{3}$

${ }^{1}$ Department of Pathology, State University of New York Upstate Medical University, Syracuse, New York, USA

2 Department of Gastroenterology, United Health Services Hospitals, Johnson City, New York, USA

${ }^{3}$ Department of Pathology and Laboratory Medicine, United Health Services Hospitals, Johnson City, New York, USA

\section{References}

${ }^{1}$ Capella C, Solcia E, Sobin LH, Arnold R. Endocrine tumors of the colon and rectum. In: Hamilton SR, Aaltonen AA (eds). World Health Organization classification of tumours. Pathology and genetics. Tumours of the digestive system.Lyon: IARC 2000: 137-139

${ }^{2}$ Gaffey MJ, Mills SE, Lack EE. Neuroendocrine carcinoma of the colon and rectum. A clinicopathologic, ultrastructural and immunohistochemical study of 24 cases. Am J Surg Pathol 1990; 14: 1010 - 1023

${ }^{3}$ Riddell RH, Petras RE, Williams GT, Sobin LH. Epithelial neoplasia of the intestines. In: Riddell RH, Petras RE, Williams GT, Sobin LH (eds). Atlas of tumor pathology. Tumors of the intestines. Washington, DC: Armed Forces Institute of Pathology 2002: 85-240

${ }^{4}$ Trecca A, Fujii T, Kato Set al. Small advanced colorectal adenocarcinomas: report on three cases. Endoscopy 1998; 30: $493-495$
${ }^{5}$ Minamoto T, Masayoshi M, Ogino Tet al. Early invasive colorectal carcinomas metastatic to the lymph node with attention to their nonpolypoid development. Am J Gastroenterol 1993; 88: $1035-1039$

\section{Corresponding Author}

\section{S. Mukhopadhyay, M.D.}

Department of Pathology State University of New York Upstate Medical University 750 East Adams Street

Syracuse, NY-13210

Fax: $\quad+1-315-464-7130$

E-mail: mukhopas@upstate.edu 